\title{
Exclusive breast-feeding promotion among HIV-infected women in South Africa: an Information-Motivation-Behavioural Skills model-based pilot intervention
}

\author{
Emily L Tuthill ${ }^{1, *}$, Lisa M Butler ${ }^{2}$, Jennifer A Pellowski ${ }^{3}$, Jacqueline M McGrath ${ }^{4,5}$, \\ Regina M Cusson ${ }^{4}$, Robert K Gable ${ }^{6}$ and Jeffrey D Fisher ${ }^{2}$ \\ ${ }^{1}$ School of Nursing, University of California, San Francisco, 2 Koret Way, San Francisco, CA 94 143, USA: ${ }^{2}$ Institute \\ for the Collaboration on Health, Intervention and Policy, University of Connecticut, Storrs, CT, USA: ${ }^{3}$ Department of \\ Psychiatry and Human Behavior, The Warren Alpert Medical School, Brown University, Providence, RI, USA: ${ }^{4}$ School \\ of Nursing, University of Connecticut, Storrs, CT, USA: ${ }^{5}$ Connecticut Children's Medical Center, Institute for Nursing \\ Research and Evidence-Based Practice, Hartford, CT, USA: ${ }^{6}$ Center for Research and Evaluation, College of Arts \& \\ Sciences, Johnson \& Wales University, Providence, RI, USA
}

Submitted 17 June 2016: Final revision received 23 November 2016: Accepted 25 November 2016: First published online 8 February 2017

\begin{abstract}
Objective: Exclusive breast-feeding (EBF) provides optimal nutrition for infants and mothers. The practice of EBF while adhering to antiretroviral medication decreases the risk of mother-to-child transmission of HIV from approximately $25 \%$ to less than 5\%. Thus the WHO recommends EBF for the first 6 months among HIV-infected women living in resource-limited settings; however, EBF rates remain low. In the present study our aim was to design and implement a pilot intervention promoting EBF among HIV-infected women.

Design: The Information-Motivation-Behavioural Skills (IMB) model was applied in a brief motivational interviewing counselling session that was tested in a small randomized controlled trial.

Setting: Pietermaritzburg, South Africa, at two comparable rural public health service clinics.

Subjects: Sixty-eight HIV-infected women in their third trimester were enrolled and completed baseline interviews between June and August 2014. Those randomized to the intervention arm received the IMB-based pilot intervention directly following baseline interviews. Follow-up interviews occurred at 6 weeks postpartum.

Results: While not significantly different between trial arms, high rates of intention and practice of EBF at 6-week follow-up were reported. Findings showed high levels of self-efficacy being significantly predictive of breast-feeding initiation and duration regardless of intervention arm.

Conclusions: Future research must account for breast-feeding self-efficacy on sustaining breast-feeding behaviour and leverage strategies to enhance self-efficacy in supportive interventions. Supporting breast-feeding behaviour through programmes that include both individual-level and multi-systems components targeting the role of health-care providers, family and community may create environments that value and support EBF behaviour.
\end{abstract}

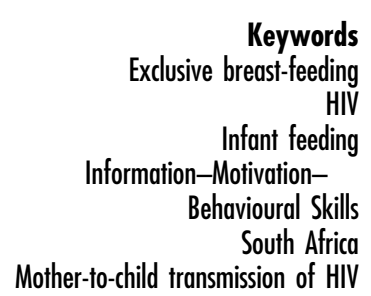

Health benefits from breast-feeding positively impact mothers and provide unparalleled benefit for their infants, including reductions in morbidity ${ }^{(1-3)}$ and improved survival $^{(1)}$. Currently, the WHO and UNICEF recommend breast-feeding initiation within the first hours after birth; exclusive breast-feeding (EBF; i.e. feeding only breast milk and no other fluids or foods) for the first 6 months; and continued breast-feeding for up to 2 years or more ${ }^{(4)}$. $\mathrm{EBF}$ is also recommended for HIV-infected women living in resource-limited settings ${ }^{(5)}$. Among women on antiretroviral therapy (ART), current evidence shows that EBF with ART reduces the risk of HIV infection while increasing HIV-free infant survival compared with mixed-feeding (i.e. feeding breast milk and other foods or fluids) ${ }^{(6)}$. Specifically, during the postpartum period, ART coupled with EBF practice can reduce mother-to-child transmission of HIV from $25 \%$ to less than $5 \%^{(7)}$ with some studies showing this decrease to less than $2 \%$. Given the benefits of breast-feeding and the 
negative consequences from mixed-feeding in resourcelimited settings, the WHO recommends that all women living with HIV in resource-limited settings exclusively breast-feed their infants for the first 6 months of life ${ }^{(5)}$.

Despite these recommendations, in South Africa, EBF up to 6 months is uncommon. The South Africa Department of Health reports only $8 \%$ of all women are continuing to exclusively breast-feed at 6 months ${ }^{(8)}$. In the context of research studies conducted in South Africa, EBF rates among those in the intervention arms are reported as $10 \%$ at 6 weeks in PROMISE-EBF ${ }^{(9)}$ and $28 \%$ at 28 weeks in the Goodstart Cohort ${ }^{(10)}$.

HIV stigma, HIV disclosure between partners, cultural norms surrounding breast-feeding and previous infant feeding experiences are all reported challenges to adopting $\mathrm{EBF}^{(11-14)}$. For example, among our target population in KwaZulu-Natal, South Africa, breast-feeding is the cultural norm; however, the introduction of other foods (e.g. porridge) or water before 6 months from other caregivers is common. Increased efforts promoting EBF through media campaigns and initiatives at the clinic level have occurred. However, critical elements that affect levels of breast-feeding determinants, including breast-feeding information, motivation and behavioural skills using a theory-based intervention, are lacking.

\section{Theoretical framework}

The current study used the Information-MotivationBehavioural Skills (IMB) model ${ }^{(15)}$ as its theoretical framework to promote EBF. The IMB model targets information, motivation and behavioural skill factors that are conceptually and empirically related to HIV risk and preventive behaviours (see Fig. 1) ${ }^{(15)}$ and has been effectively tested in research among diverse populations worldwide ${ }^{(16,17)}$.

The IMB model has three main assumptions. First, individuals require accurate prevention information regarding the behaviour change in focus (e.g. know that EBF reduces the risk of infant death due to diarrhoeal and other communicable diseases and that the risk of HIV transmission increases from mixed-feeding). Second, individuals must be motivated to engage in the target health behaviour (e.g. possess positive attitudes towards EBF and have crucial normative support for EBF practice). Third, with appropriate information and motivation, individuals will engage the behavioural skills (e.g. those skilled behaviours necessary to effectively practise EBF over time, even in challenging circumstances, which may include breast-feeding in public or among family, identifying and overcoming breast health issues, or avoiding feeding their infant water or other foods and fluids) to achieve the target behaviour (in this case, EBF).

We developed an IMB model-based pilot intervention to promote EBF. We hypothesized that mothers who were well informed, motivated and possessed the requisite behavioural skills due to an IMB model-based intervention would be more likely to initiate and maintain EBF compared with those who received only standard antenatal care. Our objective in the present paper is to show results of the impact of our pilot intervention and the feasibility of its design.

\section{Methods}

\section{Trial design}

The study took place over the time period of March 2014 through February 2015. Using a randomized controlled trial design to evaluate feasibility and the potential impact of the pilot intervention designed to improve EBF, the pilot intervention was delivered during the third trimester of pregnancy and follow-up occurred at the infant's 6-week immunization visit. Foods and fluids are often introduced within the first weeks of the infant's life ${ }^{(18)}$ and the high rate of attendance (e.g. $98 \%$ in South Africa) ${ }^{(19)}$ at the 6-week immunization visit offered both a useful snapshot of early EBF practice in the setting of a pilot randomized controlled trial and a feasible opportunity for follow-up that facilitated high rates of return.

\section{Procedure}

The pilot intervention compared standard prevention of mother-to-child transmission of HIV care with the IMB model-based intervention, which constituted an 'enrichment' of standard of care. Participants randomized to the IMB model-based intervention arm received additional support through an individual counselling session that focused on enhancing IMB breast-feeding determinants among HIV-infected women to EBF utilizing motivational interviewing techniques (details are discussed below).

\section{Recruitment}

Clinic staff referred eligible women to one of our two trained female research assistants who described the study and screened interested women for inclusion. Women who met criteria were invited to participate, which consisted of being interviewed at baseline and at 6 weeks postpartum. Those randomized to the intervention arm received a semi-brief ( $45 \mathrm{~min}$ ), one-time, tailored, one-on-one motivational interviewing counselling session with a trained female counsellor concerning infant feeding in the context of HIV. Intervention sessions were conducted after baseline interviews in a private room at the clinic. Given the physical and time-sensitive constraints of our target population of women in their third trimester, the intervention was delivered directly after the baseline interview. Participants were compensated R50 (approximately \$US 5) for participating in the study.

\section{Participants}

Study eligibility included: (i) being 18 years or older; (ii) being a woman infected with HIV; (iii) being pregnant in her third trimester (28-42 weeks); (iv) currently taking ART; and (v) planning on returning to the clinic for her infant's 6-week 
immunization visit. Participants were followed up by one of our research assistants at 6 weeks postpartum.

\section{Study setting}

The study was conducted at two comparable public health service clinics in Sweetwaters and Edendale townships, each located outside Pietermaritzburg, KwaZulu-Natal, South Africa. Pietermaritzburg and its surrounding townships has an estimated population of $500000^{(20)}$. Informal (makeshift camps, temporary housing structures) and formal housing, high population density and high rates of unemployment characterize the townships. HIV prevalence in KwaZulu-Natal Province is estimated at $16.9 \%^{(21)}$ and the infant mortality rate in South Africa for under-5 children is estimated as 47 per 1000 live births ${ }^{(22)}$.

\section{Sample size}

Based on published reports ${ }^{(18)}$, we expected EBF at 6 weeks to be $10 \%$ in the absence of the IMB intervention and we expected the IMB intervention to have a medium standardized effect ( $\mathrm{OR}=1 \cdot 8$, per Cohen). For the present pilot, a target sample size of sixty-eight eligible participants was planned (IMB intervention, $n$ 34; control, $n$ 34) to detect a medium effect size ( $7 \%$ increase in EBF over the control condition) with $80 \%$ probability of finding an effect.

\section{Randomization}

Randomization occurred using a 2-2 blocking factor generated by the study's statistician. The research assistants, who were blinded to assignment, allocated the women to intervention $v$. control arm.

\section{Outcome measures}

The primary outcome measures for pilot intervention evaluation were levels of breast-feeding information, motivation and behavioural skills and actual breast-feeding behaviours at 6 weeks postpartum.

\section{Measurements}

Participants were interviewed by one of the study's research assistants whose first language was isizulu. To measure infant feeding information, motivation and behavioural skills we used two existing tools, including the Iowa Infant Feeding Attitudes Scale (IIFAS) ${ }^{(23)}$ and the Breastfeeding Self-Efficacy Short-Form (BSES-SF) ${ }^{(24)}$, and additional items. Cross-cultural adaptation of our compiled instrument, including translation and content validity, was completed and published separately ${ }^{(25)}$.

\section{Iowa Infant Feeding Attitudes Scale}

The IIFAS has been used extensively in breast-feeding studies and consists of seventeen items using a 5-point Likert-type scale that can predict the choice of infant feeding method as reflected by measures of behavioural intentions, and the actual feeding behaviour and duration of behaviours. Reports show adequate predictive validity and internal consistency of the data, with Cronbach's $\alpha$ ranging from 0.79 to $0.86^{(23)}$. These items were used to assess breast-feeding information and motivation.

\section{Breastfeeding Self-Efficacy Short-Form}

The BSES-SF is a refined version of the larger BSES and includes fourteen items ${ }^{(24)}$. The BSES-SF was developed to measure mothers' breast-feeding self-efficacy (i.e. their perceived ability to perform breast-feeding). The BSES-SF has been tested among breast-feeding mothers antenatally and postpartum. These items were used to assess behavioural skills (including self-efficacy).

\section{Additional item development rationale and process}

Additional item development was completed for two reasons: first, to fully capture our motivation construct; and second, to ensure items spanned infant feeding content in the context of HIV. Specifically, the IIFAS and BSES-SF focus on infant feeding information, attitudes and self-efficacy. However, by assessing only information, attitudes and selfefficacy they fail to adequately measure motivation, which according to our conceptual model is comprised of attitudes and social normative support. Furthermore, social norms and attitudes in the context of HIV are unique (e.g. perceptions of community support for EBF and stigma associated with it) and require a modified approach in items targeted to non $\mathrm{HIV}$-infected women. Therefore five social norm items in the context of HIV were developed, following the item development techniques outlined by McCoach et al. ${ }^{(26)}$.

\section{Exclusive breast-feeding intentions and behaviour}

In addition, participants answered three items on their infant feeding intentions at the pre-test and ten items on actual infant feeding behaviour at the post-test. Infant feeding behavioural items included asking about current breastfeeding behaviour (e.g. EBF, breast-feeding and feeding other foods or fluids, or exclusively formula-feeding). To validate this response and account for response bias, items on what and when other foods or fluids were first introduced, in addition to a series of items assessing $24 \mathrm{~h}$ recall on their infant's intake, were included.

\section{Motivational interviewing counselling session}

Our IMB model-based intervention was delivered using motivational interviewing techniques ${ }^{(27)}$. Motivational interviewing uses an empirically tested counselling technique to deliver brief one-on-one counselling sessions with the aim of reducing ambivalence towards a particular behaviour while acting as an effective means to convey information, motivation and behavioural skills content to HIV-infected populations to change high-risk behaviour. The facilitator attended a two-day training conducted by the 
principal investigator and was also previously trained as an HIV counsellor. The facilitator began the intervention session by asking the participant how she was feeling about her pregnancy and decisions regarding infant feeding. Motivational interviewing techniques, including reflection, were employed to reduce ambivalence towards IMB deficits as discussed by the participant. Specifically, the facilitator used active listening to reflect back to the client what she had shared. Reflecting statements back to the participant stimulated further discussion surrounding concerns, questions and strategies to effectively initiate and maintain EBF practice.

\section{Control condition}

As part of routine care all participants received standard of care procedures, which typically included a brief presentation by a counsellor or other health-care provider to the group of pregnant women in the waiting room prior to any client being seen by the antenatal care staff. Content included prenatal and postnatal expectations, diet, delivery options and the promotion of EBF to all women, including those living with HIV.

\section{Statistical analyses}

Chi-square tests and $t$ tests were performed to determine if there were any differences at baseline between participants randomized to the IMB model-based intervention and standard of care control condition. Dependentsamples $t$ tests were performed to measure any significant changes in IMB determinants within condition and independent-samples $t$ tests were conducted to determine if there were significant differences between the two conditions on IMB determinants. Cohen's $d$ effect sizes for all $t$ tests were reported.

To determine predictors of EBF across all participants regardless of trial arm, bivariate regressions were conducted on the IMB construct subscales measured at baseline. Unadjusted OR are reported. Constructs that were significant at $P<0.10$ were retained and included in a multivariate regression to determine if these variables remained significant when controlling for one another. Adjusted OR are reported for variables included in the multivariate regression.

\section{Ethics approval}

Institutional review board approval was obtained from the University of Connecticut, Storrs, CT, USA and the Human Sciences Resource Council of South Africa.

\section{Results}

A total of sixty-eight participants were approached and enrolled in the study; thirty-three were randomized to our IMB model-based pilot intervention and thirty-five to the control arm. Our pilot intervention proved feasible, as illustrated in Fig. 1. Recruiting participants for the study was effective and efficient; the timing of the intervention facilitated $100 \%$ participation with adequate follow-up at 6 weeks postpartum.

All participants completed the intervention and $85 \%$ ( $n$ 58) completed the 6-week follow-up assessment. Chi-square tests and $t$ tests were performed to evaluate if any significant differences in demographics (i.e. education, employment, age, relationship status, household characteristics) existed between

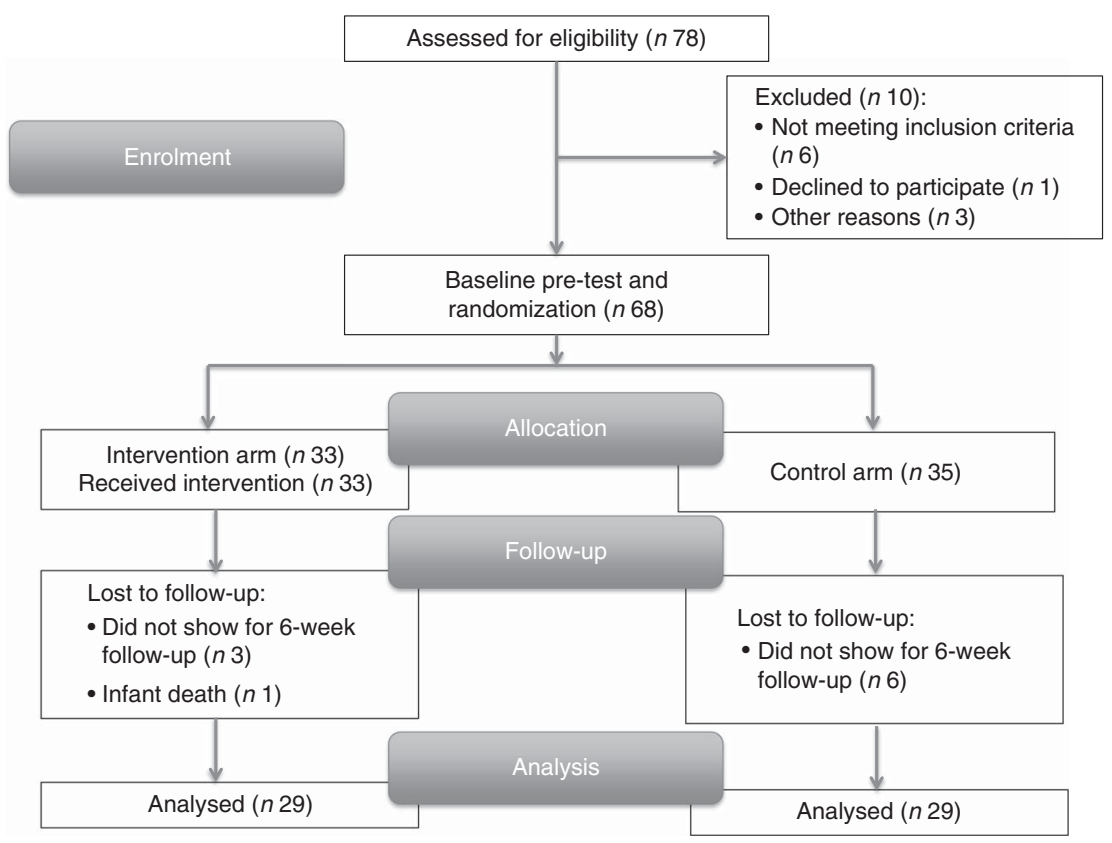

Fig. 1 Flow of participants in the randomized controlled trial to evaluate the feasibility and potential impact of a pilot intervention designed to promote exclusive breast-feeding in Pietermaritzburg, KwaZulu-Natal, South Africa 
mothers who completed the study and those who dropped out. We found no significant differences between participants who completed the 6-week follow-up and those who did not.

Demographic characteristics revealed no significant differences in between participants in the intervention and control arms (Table 1). The average age of women was 28 years and the majority had some secondary education (98\%). Most women (68\%) reported being unemployed. Ninety-six per cent of women reported having a boyfriend or partner and 36\% reported living with their mother. At the time of the study South Africa was operating under
Option B where women who attend the antenatal clinic are given ART, which they take throughout pregnancy and while breast-feeding. In addition, their infant takes nevirapine throughout the breast-feeding period. At time of follow-up all women reported receiving ART and all infants received nevirapine.

Primary information, motivation and behavioural skills breast-feeding outcomes

Regarding behavioural intention for $\mathrm{EBF}$, at their thirdtrimester antenatal appointment $87 \%$ of participants

Table 1 Participant characteristics according to study arm; HIV-infected women ( $n$ 68), Pietermaritzburg, KwaZulu-Natal, South Africa, June-August 2014

\begin{tabular}{|c|c|c|c|c|c|c|}
\hline \multirow[b]{2}{*}{ Characteristic } & \multicolumn{2}{|c|}{ IMB intervention arm $(n 33)$} & \multicolumn{2}{|c|}{ Control arm ( $n$ 35) } & \multirow[b]{2}{*}{$x^{2}$} & \multirow[b]{2}{*}{$P$} \\
\hline & $n$ & $\%$ & $n$ & $\%$ & & \\
\hline \multicolumn{7}{|l|}{ Highest level of education completed } \\
\hline Primary & 0 & 0 & 1 & 2 & & \\
\hline Some secondary & 30 & 91 & 34 & 98 & & \\
\hline Post school & 3 & 9 & 0 & 0 & $4 \cdot 195$ & 0.123 \\
\hline Currently in school & 8 & 24 & 5 & 14 & 1.089 & 0.297 \\
\hline \multicolumn{7}{|l|}{ Employment } \\
\hline Unemployed & 20 & 61 & 26 & 75 & & \\
\hline Employed & 13 & 39 & 9 & 25 & & \\
\hline Full-time & 4 & 12 & 3 & 8 & 1.452 & 0.228 \\
\hline \multicolumn{7}{|l|}{ Married } \\
\hline Boyfriend or living with as if married & 31 & 93 & 34 & 97 & 1.081 & 0.583 \\
\hline Lives with mother & 11 & 33 & 14 & 40 & 0.325 & 0.0569 \\
\hline Lives with partner's mother & 1 & 3 & 2 & 5 & 0.290 & 0.0590 \\
\hline \multicolumn{7}{|l|}{ Household characteristics } \\
\hline \multicolumn{7}{|l|}{ Drinking-water } \\
\hline Piped water from yard & 29 & 88 & 31 & 89 & & \\
\hline Piped water from public tap & 2 & 6 & 2 & 6 & & \\
\hline Tanker truck & 0 & 0 & 1 & 2.5 & & \\
\hline Rainwater & 1 & 3 & 0 & 0 & & \\
\hline Other & 2 & 6 & 1 & $2 \cdot 5$ & $2 \cdot 677$ & 0.613 \\
\hline \multicolumn{7}{|l|}{ Water treatment } \\
\hline No treatment & 31 & 92 & 32 & 91 & 0.958 & 0.619 \\
\hline \multicolumn{7}{|l|}{ Cooking fuel } \\
\hline Paraffin & 0 & 0 & 0 & 0 & & \\
\hline Gas & 2 & 6 & 2 & 6 & & \\
\hline Electricity & 31 & 94 & 29 & 83 & & \\
\hline Wood & 0 & 0 & 3 & 9 & $5 \cdot 346$ & 0.254 \\
\hline \multicolumn{7}{|l|}{ Toilet facility } \\
\hline Pit latrine & 19 & 58 & 17 & 49 & & \\
\hline Ventilated pit latrine & 11 & 33 & 16 & 46 & & \\
\hline Inside flush toilet & 2 & 6 & 1 & 2.5 & & \\
\hline Outside flush toilet & 0 & 0 & 1 & 2.5 & & \\
\hline Other & 1 & 3 & 0 & 0 & 3.314 & 0.507 \\
\hline \multicolumn{7}{|l|}{ Medical characteristics } \\
\hline \multicolumn{7}{|l|}{ Delivery location } \\
\hline Health facility & 28 & 85 & 28 & 80 & & \\
\hline On the way to a health facility & 0 & 0 & 1 & 3 & & \\
\hline Outside a health facility & 1 & 3 & 0 & 0 & & \\
\hline Other & 4 & 12 & 6 & 17 & $2 \cdot 343$ & 0.504 \\
\hline Attended postpartum & 28 & 85 & 27 & 77 & 0.693 & 0.707 \\
\hline Received antenatal counselling & 25 & 75 & 26 & 74 & 0.504 & 0.777 \\
\hline Currently on ART & 29 & 87 & 29 & 82 & 2.675 & 0.102 \\
\hline On ART at time of infant birth & 29 & 87 & 29 & 82 & 0.341 & 0.559 \\
\hline ART given during delivery & 14 & 42 & 14 & 40 & 0.410 & 0.814 \\
\hline \multirow[t]{2}{*}{ Nevirapine given to infant at delivery } & 29 & 87 & 29 & 82 & 0.341 & 0.559 \\
\hline & Mean & SD & Mean & SD & $t$ & $P$ \\
\hline Maternal age (years) & \multirow{2}{*}{\multicolumn{2}{|c|}{$25 \cdot 7-33 \cdot 8$}} & $27 \cdot 0$ & $5 \cdot 75$ & -0.739 & 0.462 \\
\hline Interquartile range & & & \multicolumn{2}{|c|}{$25 \cdot 5-33 \cdot 2^{5 \cdot 15}$} & & \\
\hline
\end{tabular}

IMB, information, motivation and behavioural skills (motivational interviewing counselling session); ART, antiretroviral therapy. 
intended to exclusively breast-feed across the two conditions and there were no significant differences in intentions at baseline (IMB intervention arm, $n 27$ (81.8\%); control arm, $n 32$ (91.4\%); $\left.\chi^{2}=1.86, P=0.39\right)$. At the 6-week postpartum follow-up, $81.5 \%$ reported EBF and there was no significant difference by trial arm (IMB intervention, $n 22$ (81.5\%); control, $n 22$ (81.5\%); $\left.\chi^{2}=0 \cdot 00, P=1 \cdot 00\right)$.

To determine if there were significant changes in EBF information, motivation and behavioural skills determinants from pre-test to post-test within trial arms, dependent-samples $t$ tests were conducted (see Table 2). For those in the intervention arm, there were no pre- to post-test significant differences in any of the IMB determinants (information, $P=0.223$; motivation (attitudes, $P=0.491$; social norms, $P=0.809$ ); behavioural skills, $P=0.707$ ). Additionally, for those in the standard of care control condition, there were also no pre- to post-test significant differences in any of the IMB determinants (information, $P=0.848$; motivation (attitudes, $P=0 \cdot 161$; social norms, $P=0 \cdot 154$ ); behavioural skills, $\quad P=0.080)$. Independent-samples $t$ tests were conducted to determine if any significant differences existed between the intervention and control conditions on IMB determinants at 6 weeks postpartum (see Table 3). Independent-samples $t$ tests showed non-significant results in the change of IMB determinants by trial arm (information, $P=0.458$; motivation (attitudes, $P=0.846$; social norms, $P=0 \cdot 266$ ); behavioural skills, $P=0.474$ ).

\section{Secondary analyses}

Because our intervention did not produce any differences in behaviour or IMB behavioural determinants of EBF behaviour, we investigated predictors of EBF behaviour to identify factors that may be influential (see Table 4). To better identify the factors driving EBF, the behavioural skills construct that was measured using the BSES-SF was divided into two groups of items made up of objective behavioural skills (e.g. 'I can always identify signs of breast health problems', 'identify a plugged duct' or 'feel confident manually expressing breast milk') and self-efficacy (e.g. 'I can always successfully cope with EBF like I have other challenging tasks') because we wanted to investigate how self-efficacy and one's belief in handling the breastfeeding environment might predict EBF itself. We left out the items that dealt with actual behavioural skills (e.g. dealing with a plugged duct, manually expressing breast milk or identifying breast health issues) given that our participants had not begun breast-feeding, were not initiating the behaviour and in effect may not relate to these items at all. Indeed, when we ran the entire BSES scale with all the items it was not significant in predicting EBF.

A bivariate regression showed EBF self-efficacy was significantly predictive of EBF behaviour (unadjusted $\mathrm{OR}=1.498 ; P=0 \cdot 016$ ). Attitudes towards EBF predicted EBF behaviour; however, it only trended on significance (unadjusted $\mathrm{OR}=1.228 ; P=0 \cdot 080$ ). Information, social norms and behavioural skills were not predictive of EBF

Table 2 Information, motivation and behavioural skills (IMB) determinants affecting exclusive breast-feeding from pre-test to post-post at 6 weeks postpartum according to study arm; HIV-infected women ( $n$ 68), Pietermaritzburg, KwaZulu-Natal, South Africa, March 2014-February 2015

\begin{tabular}{|c|c|c|c|c|c|}
\hline & \multicolumn{5}{|c|}{ Dependent-samples $t$ test } \\
\hline & $t$ & $\mathrm{df}$ & $P$ & Cohen's $d$ & SD \\
\hline \multicolumn{6}{|c|}{ IMB intervention arm ( $n$ 29) } \\
\hline Information & 1.246 & 28 & 0.223 & 0.23 & 0.19 \\
\hline \multicolumn{6}{|l|}{ Motivation } \\
\hline Attitudes & 0.699 & 28 & 0.491 & 0.13 & 0.19 \\
\hline Social norms & -0.243 & 28 & 0.809 & -0.05 & 0.19 \\
\hline Behavioural skills & -0.380 & 28 & 0.707 & -0.07 & 0.19 \\
\hline \multicolumn{6}{|l|}{ Control arm (n 29) } \\
\hline Information & 0.193 & 28 & 0.848 & 0.04 & 0.19 \\
\hline \multicolumn{6}{|l|}{ Motivation } \\
\hline Attitudes & 1.440 & 28 & 0.161 & 0.26 & 0.19 \\
\hline Social norms & 1.465 & 28 & 0.154 & 0.27 & 0.19 \\
\hline Behavioural skills & -1.816 & 28 & 0.080 & -0.34 & 0.19 \\
\hline
\end{tabular}

Table 3 Difference in information, motivation and behavioural skills (IMB) determinants between the IMB intervention and control arms at 6 weeks postpartum ( $n$ 58); HIV-infected women, Pietermaritzburg, KwaZulu-Natal, South Africa, March 2014-February 2015

\begin{tabular}{lcccrr}
\hline & \multicolumn{3}{c}{ Independent-samples $t$ test } \\
\cline { 2 - 6 } & $t$ & df & $P$ & Cohen's $d$ & 0.19 \\
\hline Information & 0.748 & 56 & 0.458 & 0.26 \\
Motivation & & 56 & 0.846 & -0.05 & 0.26 \\
$\quad$ Attitudes & -0.196 & 56 & 0.266 & -0.29 & 0.26 \\
$\quad$ Social norms & -1.123 & 56 & 0.474 & 0.19 & 0.26 \\
Behavioural skills & 0.721 & & & & \\
\hline
\end{tabular}


Table 4 Predictors of exclusive breast-feeding among HIV-infected women ( $n$ 68), Pietermaritzburg, KwaZulu-Natal, South Africa, March 2014-February 2015

\begin{tabular}{|c|c|c|c|c|c|c|}
\hline & \multicolumn{3}{|c|}{ Bivariate regression } & \multicolumn{3}{|c|}{ Multivariate regression } \\
\hline & \multicolumn{3}{|c|}{ Unadjusted } & \multicolumn{3}{|c|}{ Adjusted } \\
\hline & OR & $95 \% \mathrm{Cl}$ & $P$ & OR & $95 \% \mathrm{Cl}$ & $P$ \\
\hline Information & $1 \cdot 276$ & $0.857,1.900$ & 0.231 & - & - & - \\
\hline \multicolumn{7}{|l|}{ Motivation } \\
\hline Attitudes & $1 \cdot 228$ & $0.976,1.544$ & 0.080 & $1 \cdot 143$ & $0.893,1.463$ & 0.228 \\
\hline Social norms & $1 \cdot 190$ & $0.821,1.722$ & 0.358 & - & - & - \\
\hline \multicolumn{7}{|l|}{ Behavioural skills } \\
\hline Breast-feeding skills & $1 \cdot 198$ & $0.943,1.523$ & 0.139 & - & - & - \\
\hline Self-efficacy skills & 1.498 & $1.079,2.079$ & 0.016 & 1.425 & $1.007,2.015$ & 0.045 \\
\hline
\end{tabular}

behaviour. A multivariate regression was run to determine the individual influence of attitudes and EBF self-efficacy on predicting EBF behaviour at 6 weeks postpartum. When accounting for the variance of attitudes towards EBF, self-efficacy still remained a significant predictor of EBF (adjusted OR $=1.425 ; P=0.045$ ).

\section{Discussion}

Designing effective and sustainable interventions that result in increases in $\mathrm{EBF}$ requires approaches that take psychosocial determinants into account. In the present study we investigated whether our pilot IMB model-based intervention increased EBF determinants and behaviour per se more than receiving standard of care alone. Our results showed no difference between trial arms, perhaps due to several limitations, including positive relationships between our research assistants, possible weaknesses in our pilot intervention, participants potentiating a possible social desirability limitation and weaknesses in our compiled instrument. However, secondary analyses found breast-feeding self-efficacy to be predictive of breast-feeding initiation and EBF duration behaviours.

\section{Information-Motivation-Behavioural Skills model-based intervention}

Given the lack of variability in responses, our ability to understand the true effect of our intervention is limited. Among a different population of women with more severe IMB deficits or lower levels of reported EBF or increased sample size, our IMB model-based pilot intervention may have proven effective. However, there may have been additional factors influencing our results among our participant population.

Coinciding with the delivery of our intervention, the KwaZulu-Natal South Africa Department of Health deployed an initiative promoting $\mathrm{EBF}$ in public clinics outside Pietermaritzburg, including both study-site clinics. The initiative aimed to increase information about breast-feeding. It also provided reassurance to pregnant women about EBF in the context of HIV, often by way of health-care providers showcasing their success in decreasing rates of mother-tochild transmission of HIV within their clinic through the dual benefits of ART access to treatment and EBF practice. Many women expressed to study staff that EBF was the best practice for their infant after receiving such assurances from clinic health-care providers. Our study did not take into account these initiatives (as they were not in place at the outset of our study) nor do we know if other clinics in the area were also seeing equally high EBF rates. In these morning sessions, pregnant women received information only (which by itself has been ineffective at changing behaviours in other behavioural change breast-feeding interventions) ${ }^{(28)}$. However, it is possible that the attention to EBF may have led to an increase in its practice. Consequently, it may have also created an environment where women knew the recommended behaviour and regardless of their actual infant feeding practices reported - due to social desirability - what they knew to be the 'right answer'.

Other factors contributing to our high EBF results, in addition to the Department of Health initiative, may have been the positive and trustworthy relationships our research assistants formed with our participants. Such relationships may have resulted in a therapeutic dose among control participants, resulting in no measurable EBF behaviour differences between groups. In addition, the high levels of self-efficacy among our participants may have impacted the correspondingly high numbers of breast-feeding initiation and EBF behaviour to 6 weeks, which is reflective of assertions in the Social Cognitive Theory stating that strong self-efficacy towards a behaviour is predictive of the likelihood of successful execution of that behaviour ${ }^{(29)}$. Weaknesses in our intervention itself may also be the rationale for failing to see a difference in IMB breast-feeding determinants between conditions. However, further investigation is needed to understand the true effect of our IMB model-based intervention on impacting IMB breast-feeding determinants and EBF behaviour.

Given the objective of our pilot intervention to investigate the feasibility and potential impact of our intervention, we had some important takeaway lessons. Specifically, reaching women during their antenatal visit provided 
a time-appropriate opportunity to provide an in-depth counselling session. Given that our study design worked within the current antenatal and postpartum clinic visit schedule, our success in recruiting was high and the response from women was very positive. The feasibility of clinic staff including a 45 min counselling session with women during these visits depends on clinic resources; however, the effect on women for doing so warrants further investigation. That being said, it seems evident, especially considering other successful peer-based and postpartum-based interventions, that coupling this intervention with follow-up visits during the postpartum period is key to sustained EBF success.

\section{The role of breast-feeding determinants}

Our results showed self-efficacy being predictive of initiation and practice of EBF to 6 weeks among all participants regardless of condition. This result reinforces similar findings in the breast-feeding literature ${ }^{(30,31)}$. Individual self-efficacy items that significantly predicted breast-feeding behaviour reflected one's confidence in breast-feeding in public or around family members, being able to avoid using formula, and one's ability to adapt to any breast-feeding situation. We separated these self-efficacy items from behavioural skills items because we wanted to see how situations and one's belief in handling complex social situations (e.g. breast-feeding in public or in front of family members, etc.) predicted EBF. Behavioural skills, such as identifying a plugged duct or breast health problems, may not resonate with women prior to adapting the behaviour or practising the skill, and therefore we left these items out. Indeed, when all the BSES items were included they were not significant in predicting EBF. In this way, EBF self-efficacy items illustrate different situations that may derail breast-feeding behaviour in women with low levels of self-efficacy.

In addition to self-efficacy, other research illustrates breast-feeding knowledge as a critical determinant of successful behavioural adaption ${ }^{(32)}$, but as an isolated construct it fails to influence initiation or duration ${ }^{(28)}$. Utilizing the comprehensive approach of the IMB model accounted for this concern.

Despite our pilot intervention failing to show motivation impacting breast-feeding behaviour, previous research supports its influence ${ }^{(28)}$. However, positive attitudes towards breast-feeding by the mother can be overturned should her family undermine its practice ${ }^{(28)}$. In the context of HIV, both professional support and social support from friends and family (or lack thereof) impact infant feeding practices among woman living with HIV via cultural stigma surrounding HIV, personal beliefs and disclosure of one's status to partner and family. Thus, breast-feeding information, motivation and behavioural skills may account for important influential determinants impacting breast-feeding behaviour.

\section{Limitations}

Although our study had several strengths, limitations exist. The lack of variability in item responses limited our ability to evaluate the true impact of IMB breast-feeding determinants on EBF practice. This may be due, in part, to a lack of relevance or comprehension in our instrument despite extensive cultural adaptation procedures (see McCoach et $a l .{ }^{(26)}$ ). Furthermore, a response bias (i.e. participants self-reporting a behaviour they believe to be what the researcher is favouring regardless of whether they are practising that behaviour) may cause a falsely high EBF rate of $82 \%$ at 6 weeks postpartum among all our participants, which may be potentiated by the Department of Health initiative promoting breast-feeding. In an article by Henriques ${ }^{(33)}$, mothers' self-reports of EBF were overestimated compared with more objective information on behaviour obtained by using ${ }^{2} \mathrm{H}$ dilution techniques (a biological test using ${ }^{2} \mathrm{H}$ to detect the presence of other fluids and/or fluids in the infant's system). This may be a biological option for assessing EBF practice; however, further investigation is needed to validate its efficacy and feasibility.

In addition, due to feasibility constraints of the study, additional follow-up after 6 weeks was not possible. Obtaining these data may have revealed a greater effect of the pilot intervention on IMB determinants and breast-feeding behaviour. Furthermore, variables that were not assessed, such as when HIV diagnosis was made, previous breast-feeding experience and when breastfeeding initiation took place (e.g. time from delivery), may have provided greater insight into breast-feeding behaviour among our participant population. Finally, testing our intervention with a larger sample size may result in stronger associations between breast-feeding IMB and behaviour.

\section{Implications and future work}

In January 2015, South Africa adopted Option B+, which places pregnant women diagnosed with HIV on lifelong ART independent of CD4 count $^{(34)}$. A concern with placing HIV-infected pregnant women on lifelong therapy is the level of treatment adherence that can be maintained ${ }^{(35)}$. Treatment efficacy and access have improved greatly in South Africa, with $90 \%$ of pregnant women on effective ART during the antenatal period ${ }^{(36,37)}$. Retention in care throughout the postpartum period and beyond is fundamental to sustained treatment success (i.e. maintained viral suppression and HIV-free infant survival). Treatment adherence in the postpartum period is especially concerning given the number of barriers inherent during this intense period as a new mother. Consequently, over $50 \%$ of HIV-infected women are disengaged in HIV care by 6 months postpartum ${ }^{(37,38)}$. In the context of HIV, optimal infant health is contingent on high ART adherence in tandem with breast-feeding exclusively. Future research that supports keeping HIV-infected mothers in care postpartum is critical to keeping infants and their mothers healthy. 
Given the objective of our pilot intervention to investigate the feasibility and potential impact of our intervention, we had some important takeaway lessons. Specifically, reaching women during their antenatal visit provided a time-appropriate opportunity to provide an in-depth counselling session. Our design showed strong feasibility, with our sample ( $n$ 68) being recruited within a 2-month time frame with $100 \%$ completion of the counselling session by all those randomized to the intervention arm. Furthermore, because our study design worked within the current antenatal and postpartum clinic visit schedule, our success in recruiting was high and the response from women was very positive. The feasibility of clinic staff including a $45 \mathrm{~min}$ counselling session with women during these visits depends on clinic resources; however, the effect on women for doing so warrants further investigation. That being said, in a recently published randomized control trial conducted in KwaZulu Natal (i.e. Goodstart Cohort ${ }^{(10)}$ ), with community health workers employing motivational interviewing techniques in home visits postpartum, findings showed significant increases to EBF at 12 weeks postpartum. Future research applying our IMB model-based intervention as a packaged antenatal component to a postpartum programme (e.g. PROMISE-EBF ${ }^{(9)}$, Goodstart $\left.{ }^{(10)}\right)$ may prove useful to sustain EBF over time.

\section{Conclusions}

Although $82 \%$ of our participants reported EBF at 6 weeks postpartum, the challenges they face to execute this behaviour can be overwhelming. Encouragingly, many mothers conveyed to our research assistants that they viewed breast-feeding as the more beneficial infant feeding option and ultimately want their infant to be healthy and HIV-free. Thus, how do we best support mothers living with HIV to execute the behaviours they believe in and may even possess the confidence to perform, yet EBF behaviour at 12 weeks and beyond remains low (i.e. 8\%)? Individuallevel interventions alone may not be enough to change what has become a predominantly mixed-feeding culture in South Africa. Consequently, community-based interventions have shown some increases to EBF practice, particularly among HIV-negative women, indicating that HIV-infected women - despite being informed and supported - were not as likely to exclusively breast-feed.

Personal beliefs held by clinic and community health workers may impact counselling relationships with patients despite accurate information obtained during training. Personal experiences with infant feeding decisions made by female health-care providers may inadvertently impact their level of support for current infant feeding guidelines. For example, an HIV-infected health-care provider who used formula to feed her infant, as she may have had the resources to obtain it, may feel conflicted to recommend EBF (a different behaviour from her own) to her patients ${ }^{(39)}$. Such infant feeding experiences and beliefs may require specialized training to address bias in order to provide objective and appropriate support to HIV-infected women so that they can adhere to evidence-based guidelines. Ultimately, EBF is a complex, multifaceted, social phenomenon that is practised at the individual level, but influenced by family, community, policy makers and health-care providers. Sustaining EBF practice requires buy-in from key stakeholders at every level of influence through multi-systems, interdisciplinary interventions.

\section{Acknowledgements}

Acknowledgements: The authors thank Thandanani Children's Foundation for its assistance and support throughout the conduct of this study; and S'the Ngubane and Fiona Young for data acquisition and project management. Financial support: E.L.T. was supported by the National Institute of Mental Health/National Institutes of Health (grant number F31MH099990). The content is solely the responsibility of the authors and does not necessarily represent the official views of the National Institute of Mental Health or the National Institutes of Health. Conflict of interest: The authors declare that they have no competing interests. Authorship: E.L.T. contributed to the design, data acquisition, analysis and interpretation and writing of the manuscript. L.M.B. contributed to the analysis, content expert, instrument development and data analysis. J.A.P. assisted with analysis and writing. J.M.M. contributed to the design, manuscript development and editing. R.M.C. contributed to the design and editing of the manuscript. R.K.G. consulted on the data analysis and interpretation. J.D.F. contributed to the item development, theory-based approach and data analysis. Ethics of buman subject participation: This study was conducted according to the guidelines laid down in the Declaration of Helsinki and all procedures involving human subjects/patients were approved by the South African Human Resources Council and the University of Connecticut Institutional Review Board. Written informed consent was obtained from all subjects/patients.

\section{References}

1. Black RE, Victora CG, Walker SP et al. (2013) Maternal and child undernutrition and overweight in low-income and middle-income countries. Lancet 382, 427-451.

2. Stuebe AM \& Schwarz EB (2010) The risks and benefits of infant feeding practices for women and their children. $J$ Perinatal 30, 155-162.

3. Centre for Longitudinal Studies, UCL Institute of Education (2012) Welcome to the Millennium Cohort Study. http://www. cls.ioe.ac.uk/page. aspx? \&sitesectionid=851\&sitesectiontitle $=$ Welcome+to+the+Millennium+Cohort+Study (accessed January 2017).

4. UNICEF (2014) Breastfeeding. http://www.unicef.org/nutrition/ index_24824.html (accessed July 2016).

5. World Health Organization (2010) Guidelines on HIV and infant feeding. http://whqlibdoc.who.int/publications/ 2010/9789241599535_eng.pdf (accessed January 2017). 
6. Rollins N, Ndirangu J \& Bland R (2013) Exclusive breastfeeding, diarrhoeal morbidity and all-cause mortality in infants of HIV-infected and HIV uninfected mothers: an intervention cohort study in KwaZulu Natal, South Africa. PLoS One 8, e81307.

7. Coovadia HM, Rollins NC, Bland RM et al. (2007) Mother to child transmission of HIV-1 infection during exclusive breastfeeding in the first 6 months of life: an intervention cohort study. Lancet 369, 1107-1116.

8. Republic of South Africa, Department of Health (2003) Demographic and Health Survey 2003. Full Report. https://dhsprogram.com/pubs/pdf/FR206/FR206.pdf (accessed January 2017).

9. Tylleskar T, Jackson D, PROMISE-EBF Study Group et al. (2011) Exclusive breastfeeding promotion by peer counsellors in sub-Saharan Africa (PROMISE-EBF): a clusterrandomised trial. Lancet 378, 420-427.

10. Tomlinson M, Doherty T, Ijumba P et al. (2014) Goodstart: a cluster randomised effectiveness trial of an integrated, community-based package for maternal and newborn care, with prevention of mother to child transmission of HIV in a South African township. Trop Med Int Health 19, 256-266.

11. Chisenga M, Siame J, Baisley K et al. (2011) Determinants of infant feeding choices by Zambian mothers: a mixed quantitative and qualitative study. Matern Child Nutr 7, 148-159.

12. Doherty T, Sanders D, Jackson D et al. (2012) Early cessation of breastfeeding amongst women in South Africa: an area needing urgent attention to improve child health. BMC Pediatr 12, 105.

13. Leshabari SC, Blystad A \& Moland KM (2007) Difficult choices: infant feeding experiences of HIV-positive mothers in northern Tanzania. SAHARA J 4, 544-555.

14. Tuthill E, McGrath J \& Young S (2014) Commonalities and differences in infant feeding attitudes and practices in the context of HIV in Sub-Saharan Africa: a metasynthesis. AIDS Care 26, 214-225.

15. Fisher JD \& Fisher WA (1992) Changing AIDS risk behavior. Psychol Bull 111, 455-474.

16. Kiene S, Fisher W, Shuper P et al. (2013) Understanding HIV transmission risk behavior among HIV-infected South Africans receiving antiretroviral therapy: an informationmotivation-behavioral skills model analysis. Health Psychol 32, $860-868$.

17. Fisher JD, Cornman DH, Shuper P et al. (2014) HIV prevention counseling intervention delivered during routine clinical care reduces HIV risk behavior in HIV-infected South Africans receiving antiretroviral therapy: the Izindlela Zokuphila/Options for Health Randomized Trail. J Acquir Immune Defic Syndr 67, 499-507.

18. David S, Abbas-Chorfa F, Vanhems P et al. (2008) Promotion of WHO feeding recommendations: a model evaluating the effects on HIV-free survival in African children. J Hum Lact 24, 140-149.

19. Horwood C, Voce A, Vermaak K et al. (2010) Routine checks for HIV in children attending primary health care facilities in South Africa: attitudes of nurses and child caregivers. Soc Sci Med 70, 313-320.

20. Republic of South Africa (2012) Census (2011) http://census 2011.adrianfrith.com/place/566003 (accessed July 2016).

21. Shisana O, Rehle T, Simbayi LC et al. (2014) South African National HIV Prevalence, Incidence and Behaviour Survey, 2012.
http://www.hsrc.ac.za/uploads/pageContent/4565/SABSSM\% 20IV\%20LEO\%20final.pdf (accessed January 2017).

22. UNICEF (2015) Breastfeeding. http://www.unicef.org/ nutrition/index_24824.html (accessed October 2015).

23. De La Mora A, Russell D, Dungy C et al. (1999) The Iowa Infant Feeding Attitude Scale: analysis of reliability and validity. J Appl Soc Psychol 29, 2362-2380.

24. Dennis C (2003) The Breastfeeding Self-Efficacy Scale: psychometric assessment of the short form. J Obstet Gynecol Neonatal Nurs 32, 734-744.

25. Tuthill EL, Butler L, McGrath J et al. (2014) Cross-cultural adaptation of instruments assessing breastfeeding determinants: a multi-step approach. Int Breastfeed J 9, 16.

26. McCoach DB, Gable RK \& Madura JP (2013) Instrument Development in the Affective Domain. New York: Springer.

27. Miller WR \& Rollnick S (1991) Motivational Interviewing: Preparing People to Change Addictive Behavior. New York: Guilford Press.

28. Meedya S, Fahy K \& Kable A (2010) Factors that positively influence breastfeeding duration to 6 months: a literature review. Women Birth 23, 135-145.

29. Bandura A (1997) Self-Efficacy, The Exercise of Control. New York: Freeman and Company.

30. Thomas J, Yu EA, Tirmizi N et al. (2015) Maternal knowledge, attitudes and self-efficacy in relation to intention to exclusively breastfeed among pregnant women in rural Bangladesh. Matern Child Health 19, 47-57.

31. Wilhelm S, Rodehorst K, Stepans M et al. (2008) Influence of intention and self efficacy levels on duration of breastfeeding for Midwest rural mothers. Appl Nurs Res 2, 123-130.

32. Hector D, King L, Webb K et al. (2005) Factors affecting breastfeeding practice: applying a conceptual framework. N S W Public Health Bull 16, issue 3-4, 52-55.

33. Henriques $S$ (2015) South Africa improves exclusive breastfeeding monitoring using nuclear technique. https://www. iaea.org/sites/default/files/561058111011.pdf (accessed January 2017).

34. Republic of South Africa, Department of Health (2015) National consolidated guidelines for the prevention of mother-to-child transmission of HIV (PMTCT) and the management of HIV in children, adolescents and adults. http://www.kznhealth.gov.za/family/HIV-Guidelines-Jan2015. pdf (accessed January 2017).

35. Ngarina M, Popenoe R, Kilewo C et al. (2013) Reasons for poor adherence to antiretroviral therapy postnatally in HIV-1 infected women treated for their own health: experiences from the Mitra Plus study in Tanzania. BMC Public Health 13, 450.

36. UNAIDS (2014) South Africa. http://www.unaids.org/en/ regionscountries/countries/southafrica (accessed January 2017).

37. Hoffmann C, Coh S, Fildah M et al. (2015) Treatment failure, drug resistance, and CD4T-cell count decline among postpartum women on antiretroviral therapy in South Africa. J Acquir Immune Defic Syndr 71, 31-37.

38. Phillips T, Thebus E, Bekker L et al. (2014) Disengagement of HIV-positive pregnant and postpartum women from antiretroviral therapy services: a cohort study. J Int AIDS SOC 17, 19242.

39. Koricho A, Moland K \& Blystad A (2010) Poisonous milk and sinful mothers: the changing meaning of breastfeeding in the wake of the HIV epidemic in Addis Ababa, Ethiopia. Int Breastfeed J 5, 12. 